\title{
Large left atrial myxoma causing mitral annular dilation, functional mitral stenosis with concealed atrial septal defects
}

\author{
Jieun Song ${ }^{1}$, SooYeun Park ${ }^{1}$, Tae-Yop Kim², Tae Gyoon Yoon², and Seong-Hyop Kim² \\ Department of Anesthesiology and Pain Medicine, ${ }^{1} \mathrm{CHA}$ Bundang Medical Center, CHA University, Seongnam, ${ }^{2}$ Konkuk University \\ Medical Center, Research Institute of Biomedical Science, Konkuk University School of Medicine, Seoul, Korea
}

Transthoracic echocardiography (TTE) is a relatively precise method of evaluating intracardiac myxoma. However, if the cardiac myxoma is combined with other pathology, it could be missed by TTE. We report a case of a left atrial (LA) myxoma with mechanical mitral annular dilation and functional mitral stenosis (MS) combined with unrevealed atrial septal defects (ASD).

A 52-year-old woman was admitted to the stroke unit with aphagia and right hemiplegia. Brain magnetic resonance imaging revealed an acute infarction in the left frontotemporal area. A TTE for a stroke cardiac work-up showed a giant LA mobile mass attached to the fossa ovalis, and surgery was scheduled for removal. Anesthesia was induced and maintained using targetcontrolled infusions (Orchestra Base Primea, Fresenius Vial, Brezins, France) of propofol (target concentration 1.2-1.3 mcg/ $\mathrm{ml}$ ) and remifentanil (target concentration, 10-20 ng/ml). A preoperative TTE showed the disturbed left ventricle (LV) inflow myxoma during the diastole, with mild mitral regurgitation (MR). These findings suggested that the hemodynamic effect of the myxoma was severe MS, and we therefore carefully managed the ventricular preload and heart rate. Fluid was administered with monitoring of blood pressure, central venous pressure, pulmonary artery pressure and cardiac output, to maintain the preload, and phenylephrine was infused to prevent a decline in the systemic vascular resistance associated with remifentanil infusion. Dopamine was infused to preserve contractility. We used a transesophageal echocardiography (TEE) (X7-2t transducer and iE33; Phillips Healthcare, Andover, MA, USA) probe and evalu- ated the patient. An intraoperative TEE demonstrated that the mobile mass size was $54 \times 29 \mathrm{~mm}$ in the LA, which was attached to the fossa ovalis (Fig. 1), and revealed a secondum type of ASD (Fig. 1) with a left to right shunt. The ASD was not identified on preoperative TTE. The LA mobile mass was prolapsed across the mitral valve into the LV during the diastolic phase, partially obstructed blood flow, and caused severe functional MS. MR was also found. However, the regurgitant flow was disturbed by the myxoma, and it was difficult to quantify with two-dimensional (2D) TEE. Real-time three-dimensional (3D) TEE using the en face surgical view from the LA demonstrated that the myxoma was located centrally over the mitral valve. In addition, 3D color doppler showed a central ellipsoidal MR almost reaching the LA superior wall. Three-dimensional imaging suggested more severe regurgitation than appreciated by $2 \mathrm{D}$ imaging. An increased mitral valve annulus of $45 \mathrm{~mm}$ was observed. We noticed that the MR was significantly different from our preoperative expectations. The estimated pulmonary arterial systolic pressure was about $40 \mathrm{mmHg}$ with plethora of the inferior vena cava. We reduced the phenylephrine infusion to lower the afterload so that the forward cardiac output was maximized. The LA mobile mass was resected, including the atrial septum, resulting in enlargement of the ASD. The defect was repaired with a bovine pericardial patch. Mitral valve lifting annuloplasty via the application of a flexible strip was also performed to reduce the dilated mitral valve annulus and correct the MR. After weaning from cardiopulmonary bypass, TEE confirmed complete resection of the LA mobile mass, closure of the ASD, and a well-functioning

Corresponding author: Seong-Hyop Kim, M.D., Department of Anesthesiology and Pain Medicine, Konkuk University Hospital, 120-1, Neungdong-ro, Hwayang-dong, Gwangjin-gu, Seoul 143-729, Korea. Tel: 82-2-2030-5454, Fax: 82-2-2030-5449, E-mail: yshkim75@naver.com (c) This is an open-access article distributed under the terms of the Creative Commons Attribution Non-Commercial License (http:// creativecommons.org/licenses/by-nc/3.0/), which permits unrestricted non-commercial use, distribution, and reproduction in any medium, provided the original work is properly cited. 

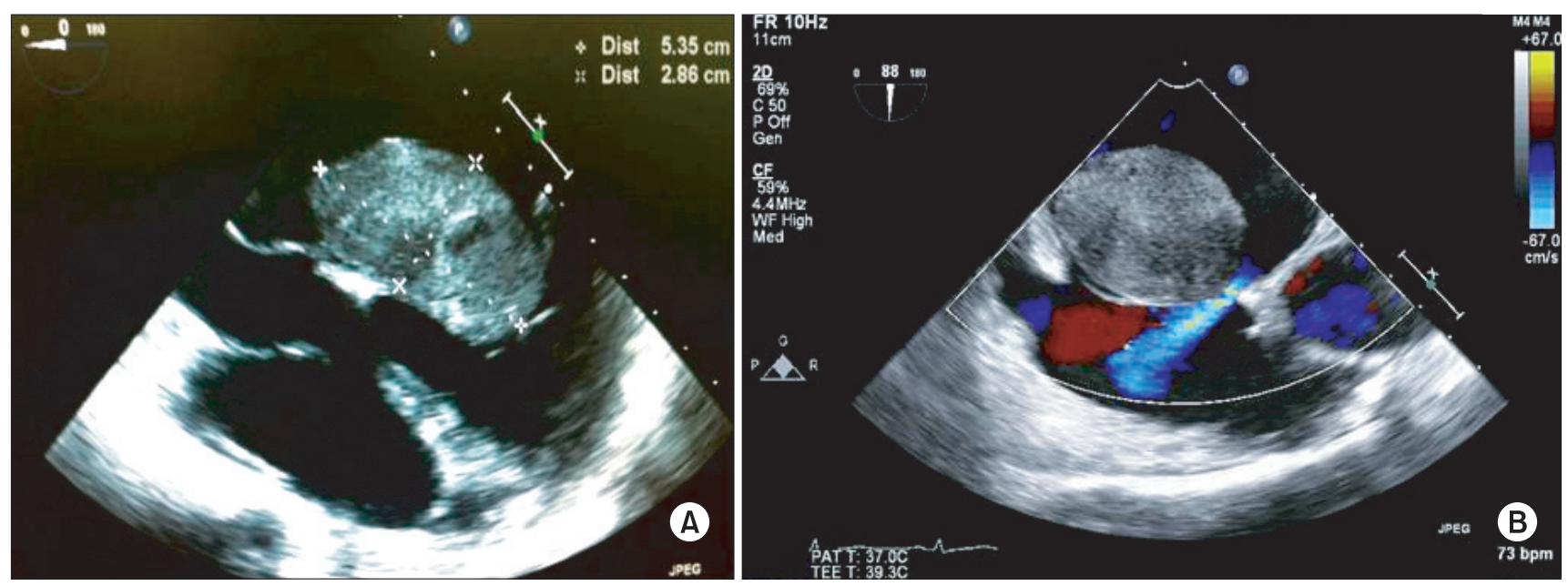

Fig. 1. (A) A mid-esophageal four-chambered view shows the prolapsed left atrial mass across the mitral valve into the left ventricle. (B) A midesophageal bicaval view with color doppler revealed the atrial septal defects.

mitral valve with a reduced mitral valve annulus of $26 \mathrm{~mm}$. The myxoma was confirmed histopathologically.

Embolism from a cardiac source accounts for $15-30 \%$ of ischemic strokes [1]. Therefore, a very detailed cardiac work-up should be considered at the first evaluation of ischemic stroke. Myxoma or ASD is one of the major risk factors for stroke. In the present case, preoperative TTE revealed only the LA mass as the embolic source, but TEE also revealed the ASD. Routine precautions concerning the influx of the air bubbles through the venous line played a vital role before we were able to diagnose ASD. Similar to the present case, concomitant occurrence of ASD must be considered in stroke patients with an atrial myxoma. A large atrial myxoma could mask the presence of a small ASD on routine TTE. Patent foramen ovale is detected in $10 \%$ of the common population and $40 \%$ of patients with stroke by contrast TTE [2].

A mitral valve inflow obstruction caused by an LA myxoma represents an important hemodynamic consequence. A myxoma disturbs LV inflow and induces elevated LA pressure and pulmonary hypertension. In this case, if the myxoma blocked the main flow, the goal of hemodynamic management is similar to that of MS management. Maintaining myocardial contractibility, preloads, and afterloads is important; keeping the heart rate stable is also essential.

The large LA myxoma could have induced MR through the mechanical annular dilation. However, the myxoma might mask the severity of concomitant MR on routine TTE. This kind of misdiagnosis precluded judicious hemodynamic management and the setting-up of an appropriate surgical plan. Matsushita et al. [3] reported a case of mitral valve annular dilatation caused by a large LA myxoma. They did not conduct an annuloplasty during the myxoma resection, as they anticipated postoperative remodeling. However, contrary to their expectation, the MR worsened after discharge and became moderately severe. Therefore, the mitral valve should be examined carefully for a myxoma prior to surgical intervention in order to lower reoperation rate. In this case, we suspected that the MR estimated preoperatively would be inaccurate, and the mitral annulus was too widely dilated to expect postoperative remodeling. We decided to combine mitral annuloplasty with myxoma excision.

Shanks et al. [4] demonstrated that 3D TEE is more accurate for quantitatively assessing both organic and functional MR as compared with 2D TEE. A large myxoma disturbs the regurgitant jet, making it difficult to evaluate regurgitant volume using 2D TEE. In such a case, 3D TEE could be valuable to estimate mitral regurgitant volume, as in the present case.

In conclusion, a high index of clinical suspicion is very important to detect intracardiac pathologies that could be missed with a preoperative TTE, or to prevent underestimation of severe complications. Intraoperative 3D TEE is also useful for evaluating MR with disturbed jet flow due to the LA mass. 


\section{References}

1. Pepi M, Evangelista A, Nihoyannopoulos P, Flachskampf FA, Athanassopoulos G, Colonna P, et al. Recommendations for echocardiography use in the diagnosis and management of cardiac sources of embolism: European Association of Echocardiography (EAE) (a registered branch of the ESC). Eur J Echocardiogr 2010; 11: 461-76.

2. Lechat P, Mas JL, Lascault G, Loron P, Theard M, Klimczac M, et al. Prevalence of patent foramen ovale in patients with stroke. N Engl J Med 1988; 318: 1148-52.

3. Matsushita T, Huynh AT, Singh T, Hayes P, Armarego S, Seah PW. Mitral valve annular dilatation caused by left atrial myxoma. Heart Lung Circ 2009; 18: 145-7.

4. Shanks M, Siebelink HM, Delgado V, van de Veire NR, Ng AC, Sieders A, et al. Quantitative assessment of mitral regurgitation: comparison between three-dimensional transesophageal echocardiography and magnetic resonance imaging. Circ Cardiovasc Imaging 2010; 3: 694-700. 